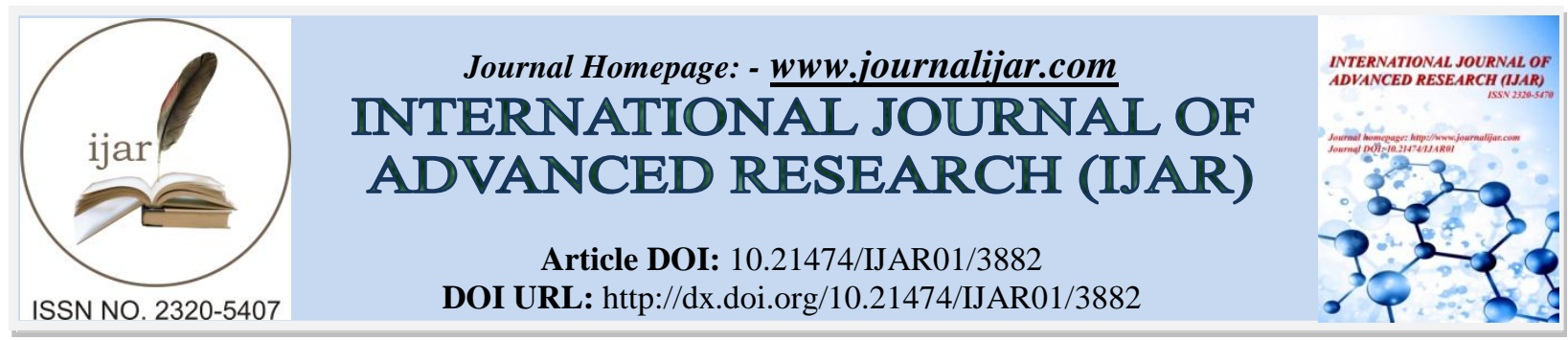

RESEARCH ARTICLE

\title{
PREVALENCE AND ANTIBIOTIC RESISTANCE PATTERN OF STREPTOCOCCUS PNEUMONIAE, ISOLATED FROM INVASIVE AND NON-INVASIVE INFECTIONS IN A TERTIARY CARE HOSPITAL OF KARACHI.
}

Fouzia Zeeshan Khan, Samina Baig, Samreen Zameer and Shaheen Sharafat. Dow University of Health Sciences, Karachi, Pakistan, Gulzar-e-Hijri, Scheme: 33, off. University Road., Karachi, Pakistan-17000.

\section{Manuscript Info}

Manuscript History

Received: 01 February 2017

Final Accepted: 08 March 2017

Published: April 2017

Key words:-

Streptococcus pneumoniae,

Antimicrobial Resistance, Invasive

Infections, Non-invasive Infections

\section{Abstract}

Objective: To determine the frequency and susceptibility pattern of multi drug resistant Streptococcus pneumoniae isolated from clinical samples of invasive and non-invasive infections.

Methods: This was a cross-sectional study conducted in the Department of Microbiology, Dow Diagnostic Reference and Research Laboratory, Karachi, Pakistan, during the period of January 2014 to October 2015. Isolation and identification of clinical isolates were performed by using standard and specific methods. Antibiotic susceptibility was performed by using Kirby Bauer method.

Results: Maximum numbers of $S$. pneumoniae were recovered from sputum, pus and blood and a higher percentage of the organisms were isolated from females. Among non-invasive $S$. pneumococcal infections, children, adolescents and adults were found to be highly resistant against Cotrimoxazole, Clindamycin and Ofloxacin, respectively. In invasive $S$. pneumococcal infections, highest resistance was observed against Penicillin G, Cotrimoxazole and Macrolides in children, adolescents and adults, respectively.

Conclusion: Emergence of antimicrobial resistance in S. pneumoniae is an alarming issue. Strict policies of infection control and surveillance programme are urgently required in order to stop the spread of antibiotic resistant pneumococci.

Copy Right, IJAR, 2017,. All rights reserved.

\section{Introduction:}

Streptococcus pneumoniae is a commensal bacterium commonly isolated from the human nasopharynx, manifesting a range of clinical infections from sinusitis, acute otitis media to meningitis, septicemia and pneumonia [1]. The burden of pneumococcal diseases is further deteriorated by large number of people suffering from chronic diseases or mycobacterial infections [2]. It is one of the main causes of high mortality and morbidity worldwide, especially for young children in developing countries [3, 4]. In developed countries, children under two years of age are more vulnerable to the pneumococcal infections [5]. An increasing trend of high mortality and morbidity in $S$. pneumoniae is directly associated with the emergence of multiple antibiotic resistance which results in misleading diagnosis and treatment failure [6]. During 1977, first time multidrug resistance against various antibiotics was detected and has been increasing steadily throughout the world [7-9]. Penicillin was the preferred treatment but as a result of evolution from susceptibility to resistance, the treatment strategies against pneumococcal infections have 
been changed. High rates of resistance against $\beta$-lactam and Macrolide have been reported in Asian countries [1012]. This situation is getting worst and adding burden on economical and medical resources. Therefore, this study was planned to determine the prevalence and antibiotic susceptibility pattern of $S$. pneumoniae isolated from different clinical sources during the period of 2014 to 2015 from a tertiary care hospital of Karachi.

\section{Methodology:}

This was a cross sectional study conducted in the Department of Microbiology, Dow Diagnostic Research and Reference Laboratory, Karachi, Pakistan, during the period of January 2014 to October 2015. The approval of study was taken from the institutional ethical and research committee.

S. pneumoniae was isolated from clinical samples including non- invasive (sputum, ear swab, eye swab and pus swab) and invasive (blood, CSF and pleural fluid). S. pneumoniae ATCC 49619 was used as a positive control.

\section{Inoculation of sample:}

Clinical specimens were inoculated on sheep blood agar (oxoid) and chocolate agar (oxoid). They further incubated in $5 \% \mathrm{CO}_{2}$ at $37^{\circ} \mathrm{C}$ for $18-24$ hours.

\section{Identification:}

Isolates of $S$. pneumoniae were identified on the basis of $\alpha$-hemolysis and inhibitory zone around optochin $(05 \mu \mathrm{g})$ disc and Gram staining.

\section{Antimicrobial testing:}

Modified Kirby-Bauer disc diffusion method was used for testing the antimicrobial susceptibility of isolates against the standards of Clinical and Laboratory Standards Institute (CLSI, 2013) [13]. Antimicrobial agents, Azithromycin $(15 \mu \mathrm{g})$, Clindamycin $(2 \mu \mathrm{g})$, Clarithromycin $(15 \mu \mathrm{g})$, Erythromycin $(15 \mu \mathrm{g})$, Penicillin $(10 \mu \mathrm{g})$, Cotrimoxazole $(1.25 / 23.7 \mu \mathrm{g})$, Ofloxacin $(5 \mu \mathrm{g})$, Linezolid $(30 \mu \mathrm{g})$ and Vancomycin $(30 \mu \mathrm{g})$ were purchased from Oxoid. Sheep Blood Agar plates were used with 0.5 Macfarland suspension and antibiotic discs were placed on them. Results were interpreted after 24 hours of incubation at $37^{\circ} \mathrm{C}$ in $5 \% \mathrm{CO}_{2}$ incubator by measuring the zones of inhibition around discs.

\section{Statistical Analysis:}

The data were analyzed using SPSS software version 20.

\section{Results:}

S. pneumoniae were isolated from the clinical samples. Out of 71 isolates, 40 were recovered from females as shown in Figure 1.

The maximum number of S. pneumoniae were isolated from the samples of Sputum (61\%) and Pus (13\%) followed by Blood (11\%), Eye Swab (6\%), Pleural Fluid (3\%), Ear Swab (3\%) and CSF (1\%) of the patients.

The resistance patterns of non-invasive pneumococcal infections against different antimicrobial agents are shown in Table 1. Highest resistance was observed against Clotrimoxazole, Clindamycin and Ofloxacin in children, adolescents and adults, respectively.

Among invasive pneumococcal infections, resistance was observed against Pencillin G, Clotrimoxazole and Macrolides in children, adolescents and adults, respectively as shown in Table 2. 
Table 1: Antibiotic Susceptibility Pattern of S.pneumoniae

\begin{tabular}{|c|c|c|c|c|c|c|}
\hline \multirow{2}{*}{ Antibiotic } & \multicolumn{2}{c|}{$\begin{array}{c}\text { Age } \\
\text { Group 1 } \\
\text { (0 to 10 years) }\end{array}$} & \multicolumn{2}{c|}{$\begin{array}{c}\text { Age } \\
\text { Group 2 } \\
(11 \text { to 20 years) } \%\end{array}$} & \multicolumn{2}{c|}{$\begin{array}{c}\text { Age } \\
\text { Group 3 } \\
\text { (>20 years) } \%\end{array}$} \\
\cline { 2 - 7 } & $\begin{array}{c}\text { Invasive } \\
\text { Infection }\end{array}$ & $\begin{array}{c}\text { Non-invasive } \\
\text { Infection }\end{array}$ & $\begin{array}{c}\text { Invasive } \\
\text { Infection }\end{array}$ & $\begin{array}{c}\text { Non-invasive } \\
\text { Infection }\end{array}$ & $\begin{array}{c}\text { Invasive } \\
\text { Infection }\end{array}$ & $\begin{array}{c}\text { Non-invasive } \\
\text { Infection }\end{array}$ \\
\hline P & 20 & 12 & 0 & 25 & 20 & 30 \\
\hline E & 20 & 13 & 0 & 38 & 40 & 33 \\
\hline CLR & 20 & 13 & 0 & 38 & 40 & 33 \\
\hline AZM & 20 & 13 & 0 & 38 & 40 & 33 \\
\hline SXT & 60 & 80 & 85 & 75 & 80 & 73 \\
\hline DA & 0 & 13 & 0 & 25 & 4 & 12 \\
\hline OFX & 20 & 13 & 0 & 13 & 13 & 18 \\
\hline LZD & 0 & 0 & 0 & 0 & 1 & 1 \\
\hline VA & 0 & 0 & 0 & 0 & 1 & 1 \\
\hline
\end{tabular}

S.pneumoniae isolated from non-invasive and invasive from different clinical samples with respect to age groups Penicillin G (P), Erythromycin (E), Clarithromycin (CLR), Azithromycin (AZM), Cotrimixazole (SXT), Clindamycin (DA), Clindamycin (OFX), Linezolid (LZD), Vancomycin( VA).

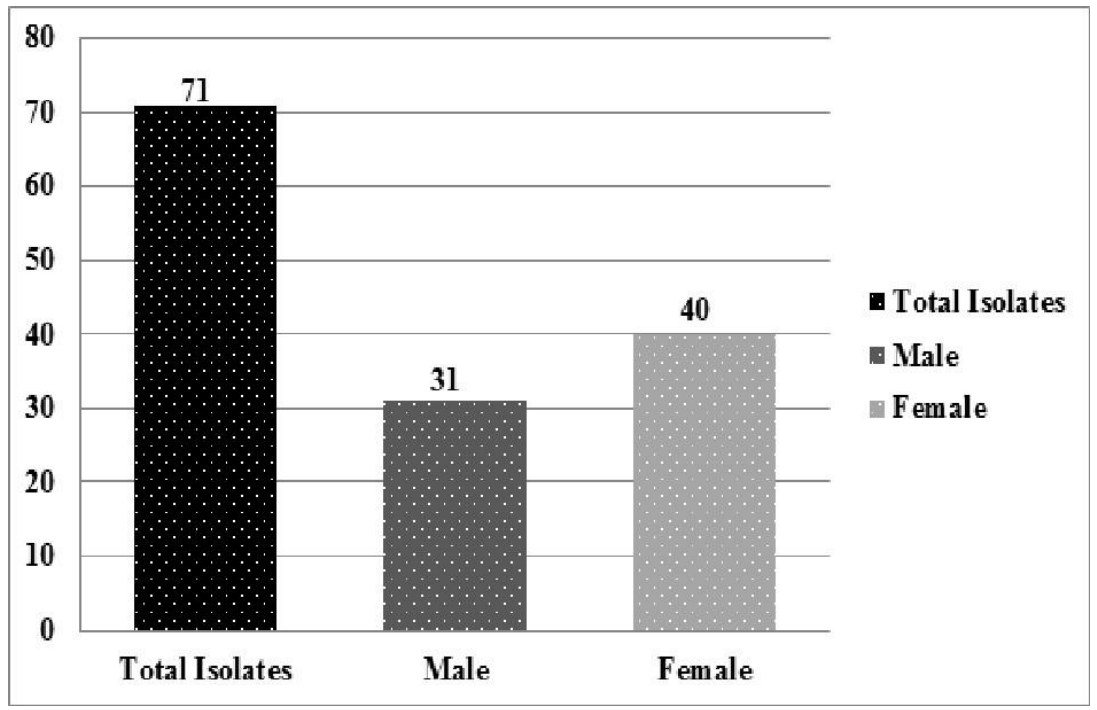

Figure 1: Distribution of S. pneumoniae between Genders 


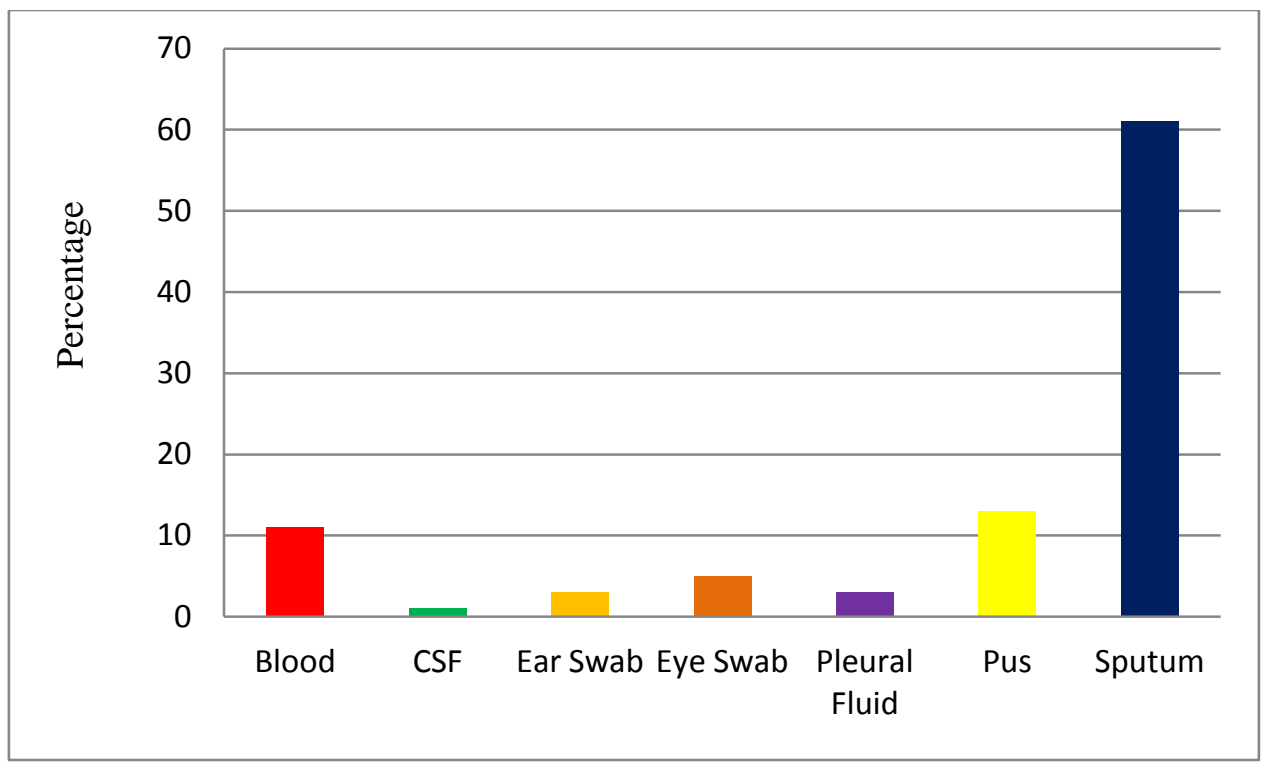

Figure 2: Frequency of S. Pneumoniae Isolated from Different Clinical Samples

\section{Discussion:}

S. pneumoniae is the leading cause of hospitalization and death of adults and children [12]. A number of significant challenges remain with regards to the diagnosis, treatment, and prevention of pneumococcal infections [6]. Treatment strategies against pneumococcal infections have been compromised by the emergence of increasing resistance of the pathogen to commonly used antibiotics [13-15]. This study discusses the prevalence and antibiotic susceptibility pattern of $S$. pneumoniae isolated from different clinical samples from a tertiary care hospital of Karachi.

Our study showed that female patients were more in number as compared to male patients suffering from pneumococcal infections, whereas, study reported male predominance [16]. Similarly, the high prevalence found in adults and in children in our study contradicted the other report [1]. Adults and children are the most vulnerable groups carrying infections due to the decreased immune profile. Pneumococci are part of normal flora of the upper respiratory tract. The organism overgrows and causes sinusitis, conjunctivitis and otitis media under favorable conditions. Pneumococcal infections are broadly divided into invasive and non-invasive infections; the former refers to infections isolated from sterile body sites. Blood borne infections occur in case of loss of integrity in the mucous membrane of the upper respiratory tract and may cause meningitis and other clinical syndromes [17].

Present study exhibited highest resistance of invasive pneumococcal infections against Pencillin G, Cotrimoxazole and Macrolides in children, adolescents and adults, respectively. Moore et al, reported Cotrimoxazole as a resistant drug in pneumococcal infections of children [18].

Several studies have reported high emergence of Penicillin and Macrolide resistance in Asian countries [19-23]. Antimicrobial resistance of $S$. pneumoniae is a global issue, more likely to be due to the over usage and improper dosage of antibiotics, over the counter sale, and malpractice of physicians. The strict implication of the drug quality and implementation of drug regulations are not easy in developing countries. Therefore, low quality of antimicrobials is increasing and equally contributing to the emergence of resistance.

B-lactams are the most commonly used antibiotics for the treatment of pneumococcal infections. Resistance against $\beta$-lactam antibiotics has developed due to the mutations in PBP (Penicillin binding proteins) [24]. The increasing resistance of $\beta$-lactams has been resulted in the high usage of non- $\beta$-lactam antibiotics for treating pneumococcal diseases. Nowadays, Macrolides are being used as an alternative treatment of choice for pneumococcal infections. Emergence of Macrolide resistance in S. pneumoniae was first reported in the early-1990s. Indiscriminate use of semi-synthetic Macrolides including Azithromycin and Clarithromycin were mainly responsible for resistance. Macrolide resistance in $S$. pneumoniae is chiefly due to methylation of ribosomes by erm (B) encoded genes, erm (B) and by a $m e f(E) m e l$ encoded efflux pump on the genetic element Mega. Current study stated $73 \%$ resistance 
against Macrolide groups, confirmed by the survey of antimicrobial resistance of Pakistan from 2002 to 2015 [25]. Resistance to Cotrimoxazole, Clindamycin and Ofloxacin was found in non-invasive pneumococcal infections of children, adolescents and adults, respectively. Cotrimoxazole resistance is also found in children suffering from upper respiratory tract infections as discussed in study [26]. Extensive use of Cotrimoxazole led to the emergence of resistance against this drug. Sulfamethoxazole-resistant isolates showed a 3 or 6 bp insertion in the Sulphonamidebinding site of folP [27]. Current study exhibited high resistance of Ofloxacin in adults but only $1.1 \%$ of Ofloxacin-non-susceptible isolates are reported, although the rate of Fluoroquinolone resistance is low in this region [28]. Development of resistance to Fluoroquinolones is due to the mutations of gyrA and parC genes that further encode type II topoisomerase subunits also called quinolone-resistance determining regions [29]. Clindamycin resistance plus macrolide resistance is a suitable indicator for detection of the erm (B) resistance marker [24]. Clindamycin resistance was reported as $22.6 \%$ coupled with a high prevalence of Macrolide resistance. These results indicated that isolates of $S$. pneumoniae most probably have erm (B) resistance marker as compared with the efflux mechanism of Macrolide resistance. The high occurrence of pneumococcal antibiotic resistance genes coupled with the ease of horizontal gene transfer have enhanced global dissemination of pneumococcal multi-drug resistance, which has significant impacts on clinical practice. Treatment guidelines for pneumococcal infections must be updated according to the changes in resistance patterns [30, 31].

\section{Conclusion:}

Our study exhibited increasing pattern of resistance in $S$. pneumoniae against commonly prescribed antibiotics. The changing trends of antibiogram of $S$. pneumoniae will help clinicians in selection of proper treatment. A continuous surveillance programme of antimicrobial resistance pattern in S. pneumoniae including both urban and rural areas are urgently required. Use of pneumococcal vaccination along with appropriate treatment is essential in order to decrease the morbidity and mortality.

\section{Abbreviations:}

PBP (Penicillin binding proteins)

\section{References:}

1. Turner P, Turner C, Jankhot A, Helen N, Lee SJ, Day NP, et al. A longitudinal study of Streptococcus pneumoniae carriage in a cohort of infants and their mothers on the Thailand-Myanmar border. PLoS One. 2012;7(5):e38271.

2. Nuorti JP, Whitney CG. Prevention of pneumococcal disease among infants and children: use of 13-valent pneumococcal conjugate vaccine and 23-valent pneumococcal polysaccharide vaccine: recommendations of the Advisory Committee on Immunization Practices (ACIP): Department of Health and Human Services, Centers for Disease Control and Prevention; 2010.

3. O'Brien KL, Wolfson LJ, Watt JP, Henkle E, Deloria-Knoll M, McCall N, et al. Burden of disease caused by Streptococcus pneumoniae in children younger than 5 years: global estimates. The Lancet. 2009;374(9693):893902 .

4. Song JY, Nahm MH, Moseley MA. Clinical implications of pneumococcal serotypes: invasive disease potential, clinical presentations, and antibiotic resistance. Journal of Korean medical science. 2013;28(1):4-15.

5. Isaacman DJ, McIntosh ED, Reinert RR. Burden of invasive pneumococcal disease and serotype distribution among Streptococcus pneumoniae isolates in young children in Europe: impact of the 7-valent pneumococcal conjugate vaccine and considerations for future conjugate vaccines. International Journal of Infectious Diseases. 2010;14(3):e197-e209.

6. Ludwig E, Bonanni P, Rohde G, Sayiner A, Torres A. The remaining challenges of pneumococcal disease in adults. European Respiratory Review. 2012;21(123):57-65.

7. Klugman KP. Pneumococcal resistance to antibiotics. Clinical microbiology reviews. 1990;3(2):171-96.

8. Jacobs MR, Koornhof HJ, Robins-Browne RM, Stevenson CM, Vermaak ZA, Freiman I, et al. Emergence of multiply resistant pneumococci. New England Journal of Medicine. 1978;299(14):735-40.

9. Azadegan A, Ahmadi A, Lari AR, Talebi M. Detection of the efflux-mediated erythromycin resistance transposon in Streptococcus pneumoniae. Annals of laboratory medicine. 2015;35(1):57-61. 
10. Song J-H, Lee NY, Ichiyama S, Yoshida R, Hirakata Y, Fu W, et al. Spread of drug-resistant Streptococcus pneumoniae in Asian countries: Asian Network for Surveillance of Resistant Pathogens (ANSORP) study. Clinical Infectious Diseases. 1999;28(6):1206-11.

11. Shibayama K, Lee H, Kim S. Comparison of antibiotic resistance rate of medically important microorganisms between Japan and Korea. Annals of Clinical Microbiology. 2015;18(4):111-8.

12. Goossens H. European strategies to control antibiotic resistance and use. Annals of Clinical Microbiology. 2014;17(1):1-8.

13. Cockerill F, Patel J, Alder J, Bradford P, Dudley M, Eliopoulos G. Performance standards for antimicrobial susceptibility testing: twenty-third informational supplement; M100-S23. Wayne, PA: CLSI. 2013.

14. Feldman C, Anderson R, editors. Antibiotic resistance of pathogens causing community-acquired pneumonia. Seminars in respiratory and critical care medicine; 2012: Thieme Medical Publishers.

15. Low DE. What is the relevance of antimicrobial resistance on the outcome of community-acquired pneumonia caused by Streptococcus pneumoniae?(Should macrolide monotherapy be used for mild pneumonia?). Infectious disease clinics of North America. 2013;27(1):87-97.

16. Steel HC, Cockeran R, Anderson R, Feldman C. Overview of community-acquired pneumonia and the role of inflammatory mechanisms in the immunopathogenesis of severe pneumococcal disease. Mediators of inflammation. 2013;2013.

17. Kim SH, Bae IK, Park D, Lee K, Kim NY, Song SA, et al. Serotype Distribution and Antimicrobial Resistance of Streptococcus pneumoniae Isolates Causing Invasive and Noninvasive Pneumococcal Diseases in Korea from 2008 to 2014. BioMed Research International. 2016;2016.

18. Bennett JE, Dolin R, Blaser MJ. Principles and practice of infectious diseases: Elsevier Health Sciences; 2014.

19. Moore CE, Giess A, Soeng S, Sar P, Kumar V, Nhoung P, et al. Characterisation of Invasive Streptococcus pneumoniae Isolated from Cambodian Children between 2007-2012. PLoS One. 2016;11(7):e0159358.

20. Kim SH, Song J-H, Chung DR, Thamlikitkul V, Yang Y, Wang H, et al. Changing trend of antimicrobial resistance and serotypes in Streptococcus pneumoniae in Asian countries: an ANSORP study. Antimicrobial agents and chemotherapy. 2012:AAC. 05658-11.

21. Xue L, Yao K, Xie G, Zheng Y, Wang C, Shang Y, et al. Serotype distribution and antimicrobial resistance of Streptococcus pneumoniae isolates that cause invasive disease among Chinese children. Clinical infectious diseases. 2010;50(5):741-4.

22. Zhao C, Zhang F, Chu Y, Liu Y, Cao B, Chen M, et al. Phenotypic and genotypic characteristic of invasive pneumococcal isolates from both children and adult patients from a multicenter surveillance in China 20052011. PLoS One. 2013;8(12):e82361.

23. Zhang Y, Chen Y, Wang Z, Li Y, Wang D, Shang Y, et al. Serological and molecular capsular typing, antibiotic susceptibility and multilocus sequence typing of Streptococcus pneumoniae isolates from invasive and noninvasive infections. Chinese medical journal. 2013;126(12):2296-303.

24. Okade H, Funatsu T, Eto M, Furuya Y, Mizunaga S, Nomura N, et al. Impact of the pneumococcal conjugate vaccine on serotype distribution and susceptibility trends of pediatric non-invasive Streptococcus pneumoniae isolates in Tokai, Japan over a 5-year period. Journal of Infection and Chemotherapy. 2014;20(7):423-8.

25. Schroeder MR, Stephens DS. Macrolide Resistance in Streptococcus pneumoniae. Frontiers in Cellular and Infection Microbiology. 2016;6.

26. Zafar A, Hasan R, Nizamuddin S, Mahmood N, Mukhtar S, Ali F, et al. Antibiotic susceptibility in Streptococcus pneumoniae, Haemophilus influenzae and Streptococcus pyogenes in Pakistan: a review of results from the Survey of Antibiotic Resistance (SOAR) 2002-15. Journal of Antimicrobial Chemotherapy. 2016;71(suppl 1):i103-i9.

27. Korona-Glowniak I, Maj M, Siwiec R, Niedzielski A, Malm A. Molecular Epidemiology of Streptococcus pneumoniae Isolates from Children with Recurrent Upper Respiratory Tract Infections. PLoS One. 2016;11(7):e0158909. 
28. Cornick JE, Harris SR, Parry CM, Moore MJ, Jassi C, Kamng'ona A, et al. Genomic identification of a novel co-trimoxazole resistance genotype and its prevalence amongst Streptococcus pneumoniae in Malawi. Journal of Antimicrobial Chemotherapy. 2014;69(2):368-74.

29. Huang S, Liu X, Lao W, Zeng S, Liang H, Zhong R, et al. Serotype distribution and antibiotic resistance of Streptococcus pneumoniae isolates collected at a Chinese hospital from 2011 to 2013. BMC infectious diseases. 2015;15(1):1.

30. Redgrave LS, Sutton SB, Webber MA, Piddock LJ. Fluoroquinolone resistance: mechanisms, impact on bacteria, and role in evolutionary success. Trends in microbiology. 2014;22(8):438-45.

31. Woodhead M, Blasi F, Ewig S, Garau J, Huchon G, Ieven M, et al. Guidelines for the management of adult lower respiratory tract infections-Full version. Clinical microbiology and infection. 2011;17(s6):E1-E59. 